\title{
A Holistic Approach to Feature-based Structural Mapping in Cross Laminated Timber Buildings
}

\author{
Mehmet Sinan Bermek ${ }^{l}$, Dennis Shelden ${ }^{2}$, T. Russel Gentry ${ }^{3}$ \\ ${ }^{1,2,3}$ Georgia Institute of Technology, School of Architecture \\ ${ }^{1}$ mbermek@gatech.edu ${ }^{2,3}\{$ dennis.shelden|russell.gentry\}@design.gatech. \\ $e d u$
}

\begin{abstract}
Mass Engineered Timber products provide a unique opportunity in configuring panelized building systems that are suitable for both prefabrication and onsite customization. The structural nature of these large section elements also brings about the need for a coordinated design-fabrication-assembly workflow. These products can assume different geometric configurations and their behaviour can be approximated globally by simplifying framing schemas. Current BIM Interoperability standards such as STEP or IFC already acknowledge and support the interconnected nature of component properties, yet these Data Models are component focused. Expanding on the relationships between components and using sets to define part to whole, or exteriority relationships could yield a more flexible and agile querying of building information. This would be a framework fit for automated feature derivation and rule based design applications. To this end Graph structures and Graph Databases, alongside existing ontology authoring tools are studied to probe new cognitive possibilities in collaborative AEC workflows
\end{abstract}

Keywords: Graph theory, BIM, CLT, IFC

The BIM paradigm has enabled breaching some of the boundaries that emerged in contemporary building practice. Projects developed within the BIM paradigm contain the information that historically required countless Requests for Information (RFI) between actors in different stages of the design and construction workflow. To name a few, the design, procurement, fabrication, transport and assembly tasks are commonly responsibilities of different agents. The intent is to encode the information into the design product and maintain it accessible among roles through central BIM repositories. In this paradigm information is accreted step by step, reducing the time and costs associated with information exchange between different actors upstream or downstream.

The documentation and modeling medium offered by BIM paradigm is still being used at best as a dynamic repository. As is, remaining bottlenecks in these mostly ad hoc workflows are solved by professionals and technicians that compile the data available by bespoke scripts, manual methods or proprietary software add-ons. These applications and aggregators are sparse and containerised at best 
leading to information loss, lack of transparency and slowing down of communication.

Our intent is to develop processing engines, schemata and active databases that can be used in automated decision making, generative design, and dynamic optimization while maintaining human readability. To this end we are focusing on Cross Laminated Timber (CLT) and other Mass Engineered Timber (MET) products, as these are large span planar elements and their internal behaviour can be reliably approximated. These properties let us develop a building model (considered as a contiguous whole) represented as a network of systems and agents with pertinent information. The possibility of having various levels of detail that can be called as needed from a central comprehensive repository is coming within reach with recent developments in cloud-connected coordination workflows and remote collaboration protocols.

CLT panels are being more frequently adopted in residential and specialised construction. The wider adoption of these products outside their vernacular region (Germany, Austria, and Northern Italy) has happened following wide scale research conducted starting from 2000's. Subsequently CLT and related connection hardware's technical specification references, alongside fire protection demands for wood materials has been included in the 2015 International Building Code (IBC). This standardisation has allowed the CLT and other MET products to be considered for larger architectural projects. Specifically their use in residential projects has sparked interest.

These products are resilient, affordable, and are unrivalled in regards of carbon sequestering compared to other construction materials adopted in contemporary buildings. Most CLT panels are prefabricated reducing jobsite waste, assembly times and costs. Other MET products like Nail Laminated Panels or Dowel Laminated Panels also provide unprecedented advantages in regards to onsite fabrication and material reuse. Experimental reuse applications include salvaging wood material from flooded forests, old wood constructions and reuse of visu- ally undesirable material in inner layers to reduce the amount of grown wood that would otherwise be destined to chipping or flaking for non structural wood products.

To this end the research proposes the development of a schema to process the BIM model and generate a Graph DataBase (GDB) to encode building component information and connectivity and store relational information in a directly accessible state. Particular attention is paid to directionality of relations between components as these relations have a key role in feature derivation, sorting, and automated part generation.

The hypothesis is that having this model will permit different actors, in different stages of design and building processes, to access, update, and verify information pertinent to diverse operations in the workflow dynamically and with a better support for automation and automated decision making.

The schema is based on the branch of discrete mathematics called graph theory which is mainly a method of representing sets and networks of relations between elements of sets. In short a graph $G$ is an ordered pair of vertices $(V)$ that represent elements of a set and edges $(E)$ that are tuples that put the aforementioned vertices in connectivity relations. Thus a Graph $\mathrm{G}$ is simply $G=\{V, E\}$ This in itself can be used to model building elements with different groupings in a scale independent continuous structure. Figure 1 shows how the same spatial relations could be represented in different graphs without contradiction.

A distinction between a GDBs and graphs in general must be made before going onward; while GDBs use graph structures for queries they are bound by limits in the level of abstraction. These databases are similar to network model databases. Most of the reasoning for the schemata will follow a more mathematical conjecture of the structural relations. Particular attention will be given to already present research threads that look into the interconnected data model nature of open BIM standards. Robust, network type, building data modeling approaches that 
Figure 1

In the two versions

of the same

assembly the

inheritance

between

connectivity that

propagates

upwards. note

these are

predefined design

domains in BIM

models that are

separated by floors.
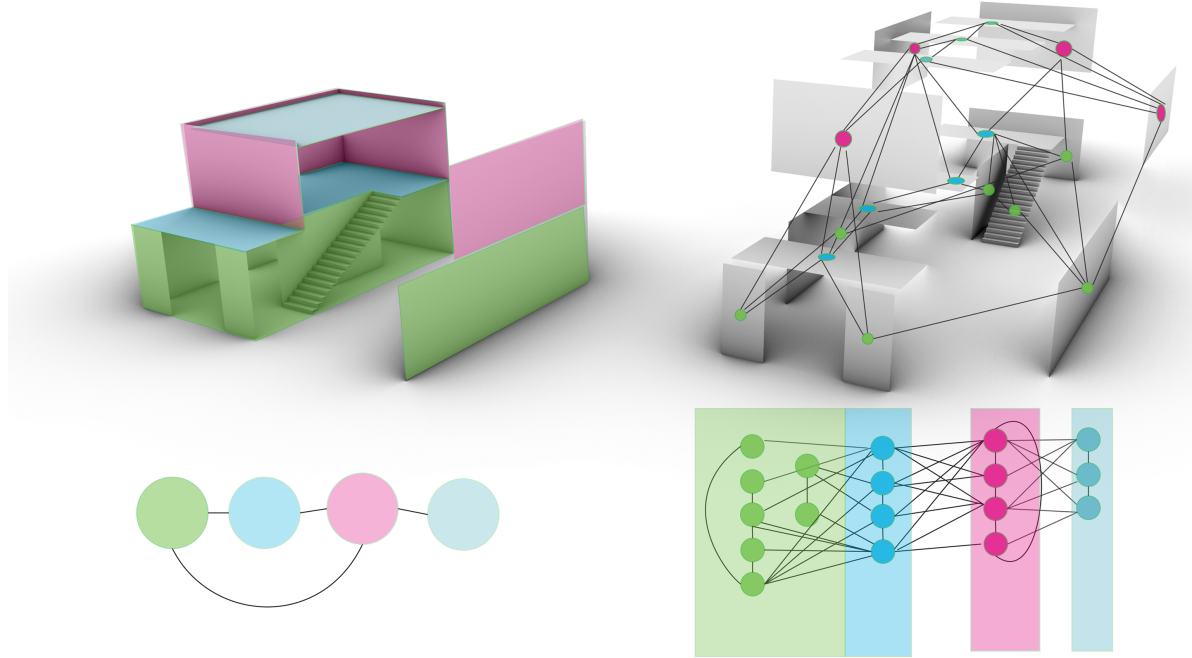

use Semantic Web Rule Language (SWRL) and Web Ontology Language (OWL) are of particular interest as these methods and approaches are already part of World Wide Web Consortium's (W3C) standards infrastructure. (Pauwels, van Deursen 2012)

There are different possibilities of defining relations between sets and elements. For the purposes of this schema only simple (as opposed to multigraphs) and directed graphs will be considered. This specification prohibits recursive relations that are found in classes as opposed to sets. I.e. a set can not contain itself as an element while it is naturally its own subset. While this kind of axiomatic specification may seem elementary most of the techniques discussed offer support for more complex multigraph realities, thus it is worth underlining that all the proposals at least in this stage will remain within the bounds simple set operations.

In Peter Chen's seminal paper in which Entity Relationship Model is proposed one very important remark is made: the real world consists of entities and relationships. The role of relationships in configuring real world objects are emphasised by placing them on par with data elements. This approach has found support in Björk's 1992 paper "A Unified Approach for Modelling Construction Information". This paper was a comparative analysis of then available AEC information models. The Object Role Models (then “Nijssen's Information Analysis Methodology (NIAM) models") plays a prominent role are discussed at length.

Both models lend themselves to a networked information model while the former has attributes for entities the latter is attribute free. These models are extremely useful in relational database instantiations ORMs are even used in graph database design and implementations. Conceptually they permit the encoding of relation typology into the high level schema of a database. Yet even Enhanced Entity Relationship derivations fall short when it comes to qualitatively define or assign variable attributes to persistent relations. The need for a proxy component or pointer to keep tally of the information causes redundancies that are without function, and reduces readability.

Current interoperability standards like ISO-STEP and IFC already assume a network like connected ap- 
proach in specifying entities. Where the relational information encoding capabilities of the schemata are insufficient IfcRelationship entities are created to contain necessary qualities when dealing with Inheritances, component breakdown etc. .
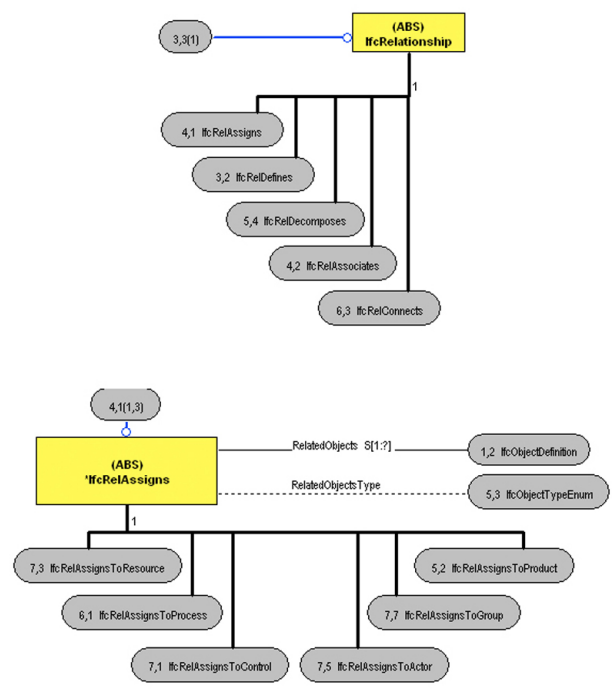

The objectified relationships in the core IFC schema are potent tools, yet information regarding proximity or assembly precedence are not encoded and would require ad hoc model view definitions (MVDs) for sharing this type of information. For example IfcReIAggregates (or IfCRelDecomposes) can contain prescriptive information regarding part to whole relationships of elements but is limited to specified component breakdown hierarchies. Pointers for spatial configuration of the elements among which relations are defined are stored within the elements itself.

Two entities are of particular interest in the IFC specifications. These are IfcRelConnects and IfCRelConnectsStructuralMember, while both are logical binders between two or more elements in the model. The former is generally used for indicating spatial continuity in case of ductwork (through abstract "port" entities) or railings it does not imply constraints if not through subtypes. These subtypes can also define applicable objects and connectivity semantics. The latter on the other hand is a perfect candidate to demonstrate the divide between application of graph algorithms and storing BIM data in Graph databases.

This is to say if through the geometric convolutions alluded at (Figure 4) we build a connected map of our structure, and we use this map to apply rulesets for detail generation and go back and store the information in a industry standard format. The nature of our working model does not necessarily need to define the output of the process. Any intersection could be mapped onto an IfcRelConnectsStructuralMember entity, with defined constrains that informs and abstract IfcStructuralConnection entity.

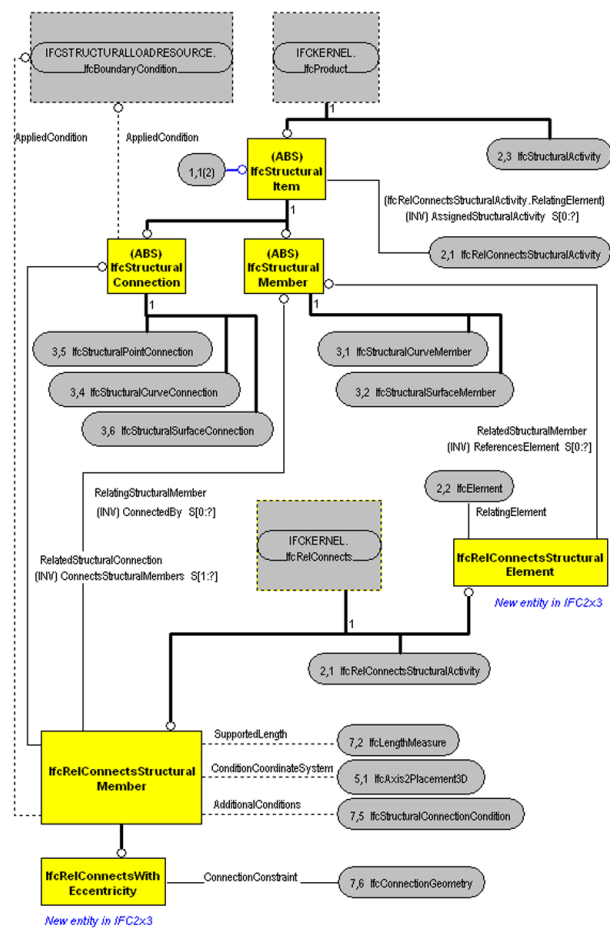

Figure 2

EXPRESS-G

SCHEMA for

IfcRelationship and IfcRelAssigns. The former is the supertype of all relationship entities in the IFC schema

Figure 3 IFC EXPRESS schema for IfcRelConnectsStructuralMember and IfcStructuralConnection 
Figure 4

Geometric Pre

Processing to

define connectivity

relations.

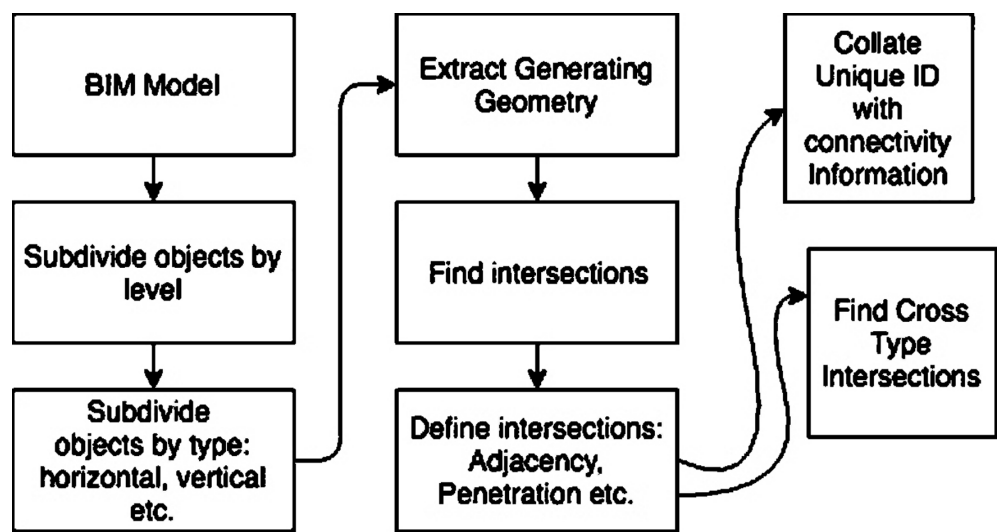

These set structures are of interest to us because the hypothesis foresees the presence of relationships and affinities that go across domain models and design stages. When stakeholder relations are established or when individual domain professionals are giving their contribute to the design process it is normal to rapidly cycle between different layers of abstraction. Especially with context during construc tion the prescribed design intent converges into a series of actions that configure material in space based on rulesets that make agents take one course of action rather than another.

The practice of subdividing objects into subcomponents that through relations maintain continuity has been in the research discourse in fields of manufacturing and computing for decades. Graph models have emerged as viable method for both visualising and probing properties of represented objects. The interconnected assemblage perspective provides us with an extensible model that will be able to populate the interfaces between components with elements generated through rule based automation, without needing to develop a new model or indexing through different stages of detailing, fabrication and assembly.

To annotate the phrase "the panels are joined by ring shank nails." on a shop drawing first of all we derive from our knowledge of fasteners, geometry and materials, secondly we provide information that is going to be interpreted by the inference mechanism of other agents. The phrase itself contains only two component references (panel, nail), one quantity information (many), one type specification (ring shank) and one relation (join); yet based on this limited specification, the architect will know there are two elements that meet and are made out of a material that can be pierced, the engineer might infer that the joint might be subject to cyclical or tension loading, the installer will be able to decide what kind of equipment is necessary to assemble the elements. (Goel 1992)

Under this light, traditional architecture tools such as hand sketches and models function not only by demonstration but by eliciting relation recall in the receiving end. (Newstetter, Eastman, \& McCracken 2001) The relational information in hand sketch line configurations have been studied in graph theory context. (Moinet, Mandil, \& Serre 2014)

One of the key reasons for which a combination of spatial and relational information plays an important role is that in most technological buildings, some components or attributes might be free of relationships of interiority. That is to say: in a building, or its model thereof, not all elements are components of other parts. This precludes the possibility of organizing component information as a hierarchical tree structure. Furthermore some actors of the building process remain agnostic even to the existence of cer- 


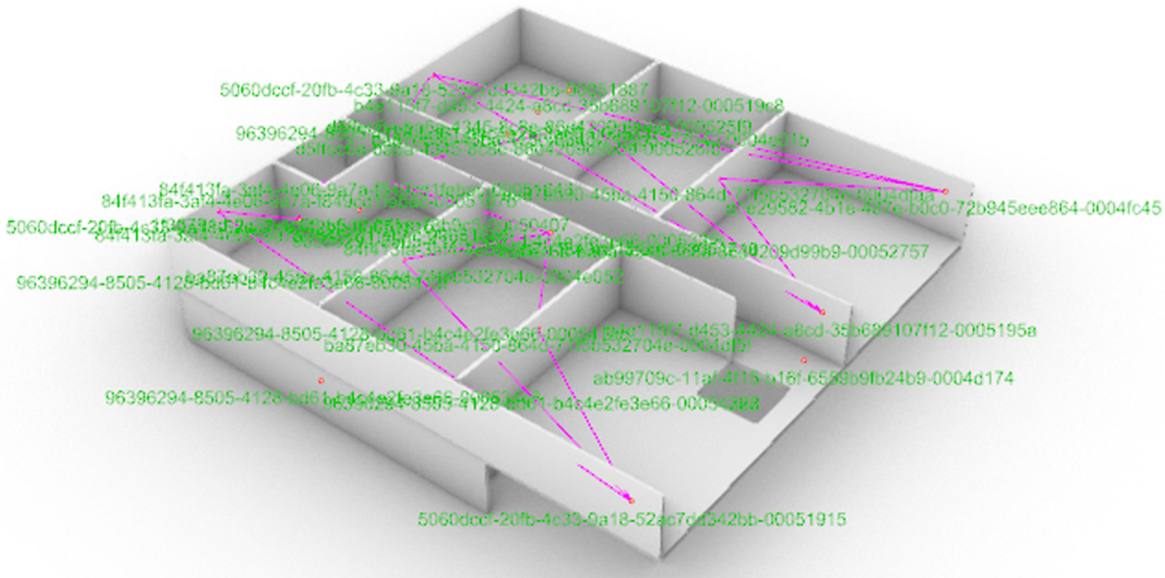

Figure 5

Connectivity map in

relation with the

Global Unique

Identifiers of

designer specified

vertical elements

before subdivision.

tain objects and systems that can play a crucial role in the whole or parts of the construction workflow.

This is especially true for connection components of the structure that are parts that populate interfaces between massive elements. While remaining out of focus for most specialities and for most of the design and fabrication stages, these can have a key role in defining the overall behaviour of the whole and geometric constraints for fabrication and assembly. This is the case of most precast or prefabricated construction elements, and Building Object Behaviour (BOB) methodology proposed by Lee et al. (2006) later applied for load bearing masonry applications by Cavieres et al. (2011) has been an important basis for defining the bottom up framework for defining final fabrication specifications.

At this point it is crucial to underline that there are already open source tools available to make an automated conversion between IFC data and GDBs. (Khalili, Chua 2015) (Tauscher, Smarsly, \& Bargstadt 2016) These yield GDB's of property relations, semantics and propose many novel querying capabilities for analysing and exploring BIM models. Yet there still is no comprehensive Framework for creating a a connectivity map or similar graph based geometry information consequently even less so for creation of new geometry based on results of geometric feature derivations and Boolean operations. Dimensionally Extended nine-Intersection Model (DE-9IM) is the most thorough standard and topological model for the solution of the spatial relations in $R^{2}$. Its partial use is going to be essential for the feature derivation. A subset matching of processed information and derivation protocols has been discussed in previous work. (Bermek, Shelden, \& Gentry 2019)

Returning Back to BOB methods, considering that our Spatial Connectivity queries are going to yield us proximity location, proximity type (be it adjacency, penetration etc.) a predefined rule set enriched by knowledge of whether the interacting entities' nature (structural, architectural) is going to yield sufficient information to inform geometric constraint types for the series of intersections. This in turn, can be fed from a project specific library of components 
for connections and inform fabrication specification modifications to initial entities to house the fasteners.

In GDBs obtained by direct conversion of IFC models has yielded successful results in information filtering and retrieval such as obtaining property sets, filtering by layer, automated retrieval of connectivity information where necessary IFC relations are part of the model. (Ismail 2017)Neo4j being an open source GDB solution is widely used in Graph building information model research. The common query language for Neo4j is Cypher it is not optimized for performance so the comparisons between traditional information retrieval systems are not in the focus of research. Yet thorough clash detection and analysis research has yielded comparable results with traditional techniques ( $\mathrm{Hu}$, Castro-Lacouture, \& Eastman 2019) still for non domain experts in IFC, and Graph databases flexible query generations solutions are not yet available.

Graph information models and by extension GDB's have the unique advantage of having proximity information embedded. Proximity and adjacency relations of components can be used to describe neighbourhoods that in turn can summarize a number of elements in component sets that can behave as a whole in the global model. Furthermore with the common model framework it would be possible to give feedback to actors upstream in the workflow without the need of new models or overhauling communication protocols. Following the example of the shop drawing annotation: some of the given notions can become part of the design in different stages the decision for the fastener type might be downstream from engineer's revision to connection details but upstream from the carpenter's tool choice. Should material or tools for complying with the specifications not be available downstream, informing the model and defining areas where the planned versus possible actions clash would add a new layer of functionality to the BIM model.

The possibility of traversing a design region to and fro between varying levels of complexity and ap- proximation yields a new cognitive horizon in collaborative AEC workflows. New generation building products like METs that provide modular solutions that also support bespoke handling of materials and component geometries will benefit extensively from the availability of a cross discipline information repository that is also stage independent, fluid and exchangeable.

\section{REFERENCES}

Bermek, MS, Shelden, D and Gentry, RT 2019 'Schema for Automated Generation of CLT Framing and Panelization', Computing in Civil Engineering 2019: Visualization, Information Modeling, and Simulation, Atlanta, pp. 312 - 319

Cavieres, A, Gentry, T and Al-Haddad, T 2011, 'Knowledge-Based Parametric Tools for Concrete Masonry Walls: Conceptual Design and Preliminary Structural Analysis', Automation in Construction, 20(6), pp. 661-740

Eastman, C, Lee, Jm, Jeong, Ys and Lee, Jk 2009, 'Automatic rule-based checking of building designs', $A u$ tomation in Construction, 18(8), pp. 1011-1033

Goel, V and Pirolli, P 1992, 'The structure of Design Problem Spaces', Cognitive Science, 16(3), pp. 395-429

Ismail, A, Nahar, A and Scherer, R 2017, 'Application of graph databases and graph theory concepts for advanced analysing of BIM models based on IFC standard', Proceedings of EGICE, -, p. -

Kudsk, A, Hvam, L, Thuesen, C, Gronvold, MO and Olsen, $\mathrm{MH} 2013$, 'Modularization in the construction industry using a top-down approach', Open Construction and Building Technology Journal, 7, pp. 88-98

Lee, g, Sacks, r and Eastman, CM 2006, 'Specifying parametric building object behavior (BOB) for building information modeling system, Automation In Construction, 15(6), pp. 758-776

Moinet, M, Mandil, G and Serre, P 2014, 'Defining tools to address over-constrained geometric problems in Computer Aided Design', Computer-Aided Design, 48, pp. 42-52

Newstetter, WC, Eastman, CM and McCraken, WM (eds) 2001, Design Knowing and Learning: Cognition in Design Education, Elsevier, Atlanta

Fenves SJ, MML 1981, 'The use of artificial intelligence techniques in preliminary structural design', Research Showcase@CMU,-, p. -

Sunil, VB, Agarwal, R and Pande, SS 2010, 'An approach to recognize interacting features from $B-\operatorname{Rep} C A D$ 
models of prismatic machined parts using a hybrid (graph and rule based) technique', Computers in Industry, 61(7), pp. 686-701

Tauscher, E, Bargstadt, HJ and Smarsly, K 2016 'Generic BIM queries based on the IFC object model using graph theory', 16th International Conference on Computing in Civil and Building Engineering, Osaka

van Treeck, C and Rank, E 2004, 'Analysis of Building Structure and Topology Based on Graph Theory', in Issa Issa, R and Flood, i (eds) 2004, 10. International Conference on Computing in Civil and Building Engineering, ASCE, Weimar, Germany, p. 10 Seiten

Weaver, W 1990, Matrix analysis of framed structures, New York: Van Nostrand Reinhold, New York

Hu Y, CLD 2019, 'Holistic clash detection improvement using a component dependent network in BIM projects', Automation in Construction, 105, p. 102832 